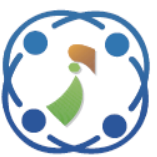

\title{
A Novel Design of Miniaturized Tag Antenna Loaded Metamaterial Spiral Split Ring Resonator
}

\author{
Abdelhadi Ennajih ${ }^{1 *}$ \\ Badr Nasiri ${ }^{1}$ \\ Jamal Zbitou ${ }^{1}$ \\ Ahmed Errkik ${ }^{1}$ \\ Mohamed Latrach ${ }^{2}$ \\ ${ }^{1}$ Laboratory of Mechanic, Energetic, Electronic and Telecommunications, Faculty of Sciences and Technics, \\ Hassan first University, Settat, Morocco \\ ${ }^{2}$ Microwave Group, Ecole Supérieure d'Electronique de l'Ouest, Angers, France \\ * Corresponding author's Email: abdelhadi.ennajih@gmail.com
}

\begin{abstract}
In this article, we propose a new design of a miniature antenna for integration into the RFID (Radio Frequency Identification) reader and the RFID transponder to operate in the Moroccan and European UHF (Ultra High Frequency) band. The proposed antenna is designed on a flexible PET (Polyethylene Terephthalate) substrate. The proposed tag consists of a microchip and a dipole antenna loaded metamaterial based on a spiral split ring resonator which has a negative magnetic permeability in the UHF RFID Moroccan and European band. The metamaterial unit cell is used to reduce the antenna size and a double T-matching structure is inserted to match the dipole antenna with the "Monza 1a" chip. The antenna yields an omnidirectional radiation patterns and a gain of 2.2 $\mathrm{dBi}$ at the central frequency. The designed antenna has a small size about $63 \mathrm{~mm} \times 14 \mathrm{~mm}$ which is suitable for paper boxes and bottles labelling.
\end{abstract}

Keywords: Tag antenna, Radio frequency identification, Metamaterial, Miniature antenna.

\section{Introduction}

Nowadays, RFID technology has become very popular [1] due to its exponential growth applications in many areas, such as: animal tracking, supply chain, distribution, access control, libraries and so on [2].

RFID is an automatic identification technology, such as voice, optical character or bar code recognition. The purpose of these technologies is to allow the identification of persons, animals or objects and the collection of information about them without human intervention. It has the distinction to operate using radio waves without line of sight or contact between the interrogator and the transponder. A RFID system includes a reader to extract information about the tagged objects, and a transponder contains information and inserted on these objects [3]. Fig. 1 presents the RFID system components.
RFID technology uses electromagnetic waves for communication between the tag and the reader. However, the different frequencies are allocated by the regulatory organizations which set very specific rules for the use of each frequency band. RFID applications use different frequency bands, including frequencies from $125 \mathrm{KHz}$ to $5.8 \mathrm{GHz}$ in the microwave range. There are several frequency bands allowed to the RFID technology such as LF band (low-frequency), HF band (high-frequency), UHF band (ultra-high-frequency) and MW band (microwave frequency) [4].

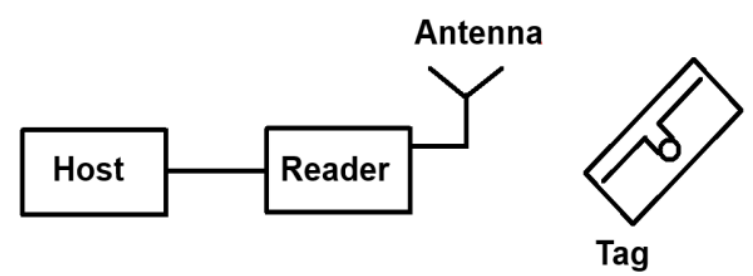

Figure.1 Basic components of an RFID system 
In recent years, RFID technology in the passive UHF band has received a great attention from researchers because of its multiple advantages in terms of size, data transfer rate and, antenna reading distance. RFID applications require tag antenna to be low cost, easy to fabricate and to integrate on the objects to be identified. The transponder is composed of an electronic chip attached to an antenna and a matching circuit is used to match the impedance of the antenna to the impedance of the RFID microchip. The electronic chip includes a basic modulation circuit and a memory. The transponder is powered by the RF (Radio Frequency) signal emitted by the reader. A variable voltage is generated at the antenna terminal when the RF (Radio Frequency) field passes through this antenna. This voltage is rectified by a PIN diode (Positive Intrinsic Negative diode) to cause a DC voltage (Direct Current) for the operation of the chip. The device becomes functional when the DC voltage achieves a certain threshold. The data stored in the microchip is then backscattered.

Passive RFID applications require antennas with small size, high gain, good read range and, low cost. RFID technology also needs flexible antennas for certain applications, such as paper boxes and bottles labelling. However, these features are difficult to achieve at the same time. Many techniques have been proposed to reduce the tag antenna size, such as fractal technology [5], the use of slots [6], the meander line technique [7] and, the use of metamaterials [8].

In [5], Kimouche et al. reached a miniaturization rate of $37.2 \%$ in comparison with the conventional dipole antenna and a gain of $1.11 \mathrm{dBi}$ by using the fractal geometry. In [6], Elhamraoui et al. achieved a miniaturization of $52.42 \%$ and a gain of 1.6 by using the slot technique. The results of these two works have a good miniaturization and acceptable performance, but the antennas still have a large size which is not suitable for some RFID applications.

In [7], with the use of the meandering technique, Choudhary et al. achieved a good miniaturization rate of $91.25 \%$ compared to the conventional dipole antenna but, the antenna has a low gain about -16 dBi. In [8], with the use of metamaterials in the ground plane, Frigui et al. achieved a good miniaturization of $79 \%$ but the antenna presents a low gain of $-1.39 \mathrm{dBi}$. After studying various techniques of miniaturizations, we found that the metamaterials can further miniaturize the antenna size without more decreasing its performance. We will take advantage of metamaterials to design a miniature antenna while improving its performance.
The goal of our work is to design a miniature antenna on a PET flexible substrate loaded metamaterial unit cells to integrate it into the reader and the tag for RFID applications in the Moroccan and European UHF band. The purpose of using the metamaterial unit cell is to reduce the size of the antenna and to improve the impedance matching between the antenna and the microchip without reducing antenna performance such as gain, read range and bandwidth.

This paper is organized according to the following plan: in the second part we will present a general view of metamaterials, in the third part we will present the different design stages of the proposed antennas for the reader and the tag and, the last part will be devoted to the conclusion.

\section{Metamaterial unit cell}

Metamaterials are artificial structures or materials which present some electromagnetic properties unavailable in nature, such as simultaneously negative permittivity and negative permeability [9]. These artificial materials consist of a combination of periodic structures that behave like an homogeneous material [10]. The electromagnetic properties of metamaterials are determined by two important parameters of the material, the negative dielectric permittivity that can be obtained by an arrangement of metallic TW (Thin Wires) and the negative magnetic permeability that can be achieved by an arrangement of SRRs (Split Ring Resonators) (Fig.2) [11].

Recently, several studies have been conducted to use these materials in filters [12,13], amplifiers and antenna structures $[14,15]$ to miniaturize their sizes or to improve their performance. In this work we used a spiral SRR structure qualified by negative permeability metamaterials to miniaturize the size of a dipole antenna and to improve its performances. The dimensions and the geometry of this structure is presented respectively in Table 1 and Fig. 3.

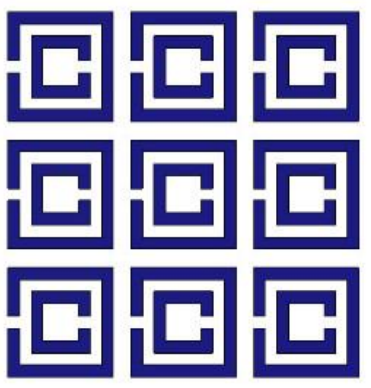

(a)

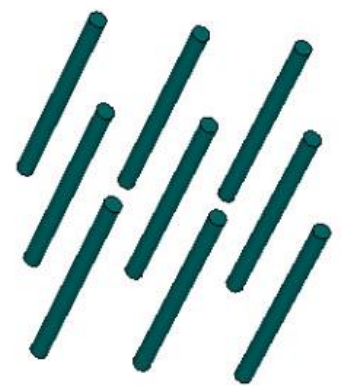

(b)
Figure.2 Metamaterial structures: (a) split ring resonators and $(b)$ thin wires 
After the design of the spiral SRR unit cell, it is integrated in a radiation box in order to find the values of its effective parameters. The electrical wall conditions are applied on the xy plane and the magnetic walls are applied on the xz plane and the propagation is along the $\mathrm{x}$ axis as illustrated in Fig.4.

The simulated S11 and S21 of the proposed metamaterial spiral SRR are presented in Fig. 5.

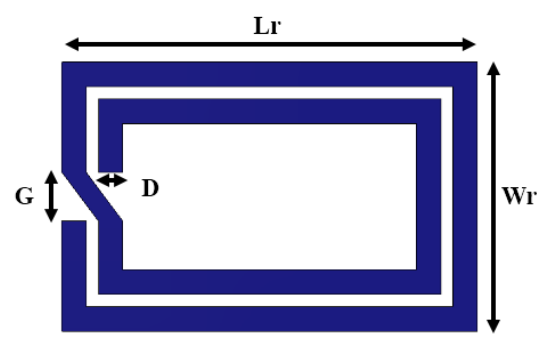

Figure.3 Proposed metamaterial structure

Table 1. Dimensions of the proposed metamaterial

\begin{tabular}{|c|c|}
\hline \multicolumn{2}{|c|}{ structure } \\
\hline Parameter & Value $(\mathbf{m m})$ \\
\hline Lr & 17 \\
\hline Wr & 11 \\
\hline G & 2 \\
\hline D & 1 \\
\hline
\end{tabular}

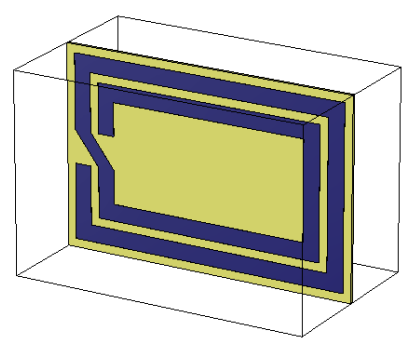

Figure.4 Proposed metamaterial unit cell in a TEM box

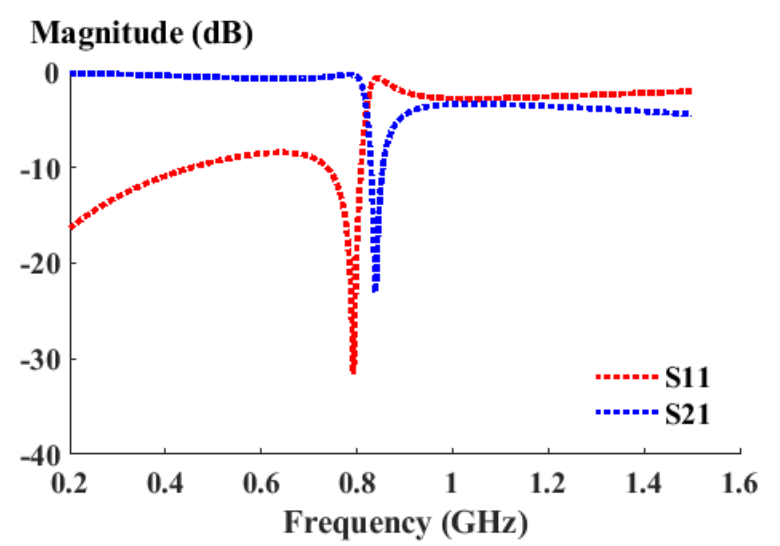

Figure.5 Simulated S-parameters of the spiral SRR
Several methods are currently used to characterize metamaterial structures from Sparameters, in this work we present the most commonly used method known as NRW (NicolsonRoss-Weir). This method will also be used in our work, it begins with the composite terms V1 and V2 given by [16]:

$$
\begin{aligned}
& V_{1}=S 21+S 11 \\
& V_{2}=S 21-S 11
\end{aligned}
$$

Where S21 is the transmission coefficient and S11 is the reflection coefficient. The formulas of permeability and permittivity are respectively given by:

$$
\begin{gathered}
\mu_{r}=\frac{2}{j K_{0} d} \cdot \frac{1-V_{2}}{1+V_{2}} \\
\varepsilon_{r}=\frac{2}{j K_{0} d} \cdot \frac{1-V_{1}}{1+V_{1}}
\end{gathered}
$$

Where $\mathrm{d}$ is the electrical thickness of the metamaterial cell, $\mathrm{K}_{0}$ is the complex wave number given by:

$$
K_{0}=\omega \frac{\sqrt{\varepsilon_{r} \cdot \mu_{r}}}{c}
$$

Fig. 6 and Fig. 7 illustrate respectively real and imaginary parts of the permeability and the permittivity computed from the $\mathrm{S}$ parameters. The permeability is negative over the frequency range between $800 \mathrm{MHz}$ and $1.1 \mathrm{GHz}$ which confirms the metamaterial effect of this structure around the desired operating band for the antenna.

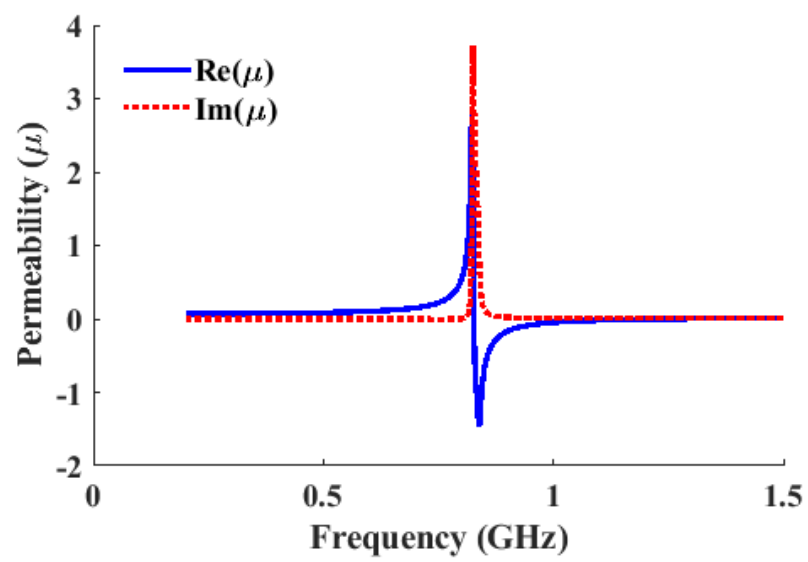

Figure.6 Calculated permeability of the proposed metamaterial unit cell 


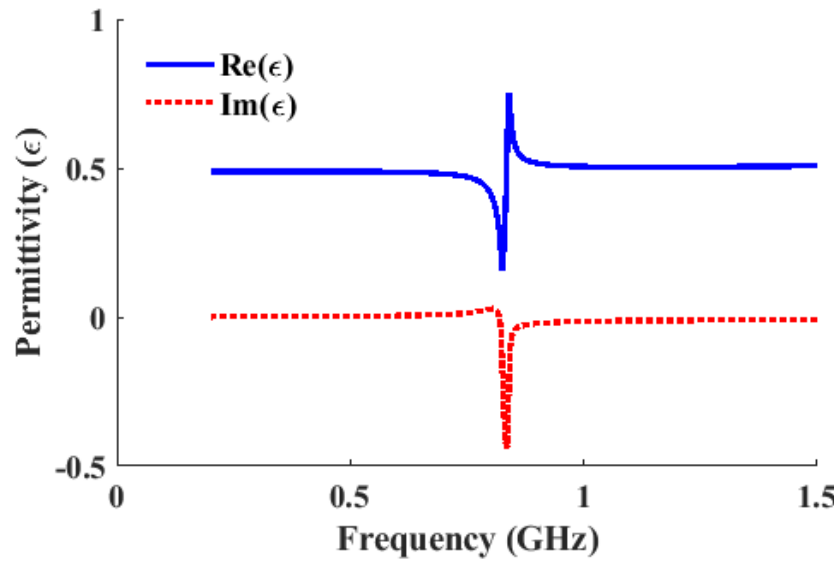

Figure.7 Calculated permittivity of the proposed metamaterial unit cell

After the design of the spiral SRR cell and the verification of the metamaterial effect, in the next sections, we will use this structure for the miniaturization of two antennas, one will be used for the RFID reader and the second one for the tag which will operate in the European and Moroccan UHF band.

\section{Reader antenna design}

\subsection{Reader antenna geometry}

The proposed antenna is based on a dipole antenna and will be integrated into an RFID reader in the UHF band around the $868 \mathrm{MHz}$. To obtain a good performance of a dipole antenna, its length must be equal to the half-wavelength, its length $\mathrm{L}$ can be calculated using the following equation [17]:

$$
L=\frac{\lambda}{2}=\frac{v}{2 f}
$$

Where $\lambda$ is the wavelength, $\mathrm{f}$ is the resonant frequency of the dipole antenna and, $v$ represents the speed of propagation in the medium, this velocity depends on the effective dielectric constant of the medium the dipole and the velocity of light, it is calculated from the following equation:

$$
v=\frac{c}{\sqrt{\varepsilon_{e f f}}}
$$

Where $c$ is the light velocity and eeff is the effective dielectric constant of the medium which is given by the following equation:

$$
\varepsilon_{\text {eff }}=\frac{\varepsilon_{r}+1}{2}+\frac{\varepsilon_{r}-1}{2}\left[1+12 \frac{h}{W}\right]^{-\frac{1}{2}}
$$

Where $W$ and $h$ are respectively the width of the dipole and the thickness of the substrate and $\varepsilon r$ its relative permittivity.

Based on the calculated results obtained for the conventional dipole antenna, we used the folding technique to miniaturize its size. In the last stage of the design, we have loaded this antenna by the metamaterial structure that we designed in the previous section. The proposed antenna has been simulated and optimized using CST (Computer Simulation Technology) software on a PET substrate with a dielectric permittivity of 3.0 and tangent loss of 0.002 , the total size of this antenna is $59 \mathrm{~mm} \times 15 \mathrm{~mm}$. Fig. 8 illustrates the design steps for the proposed antenna and the optimal geometric dimensions are shown in Table 2.

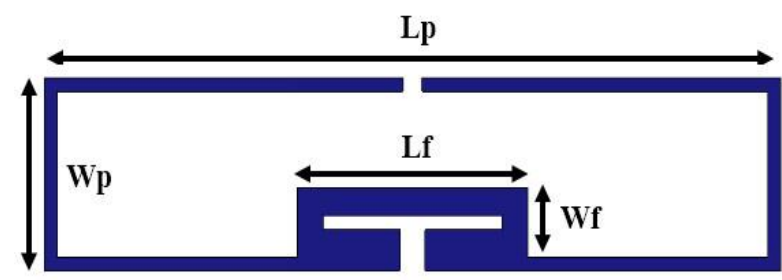

(a)

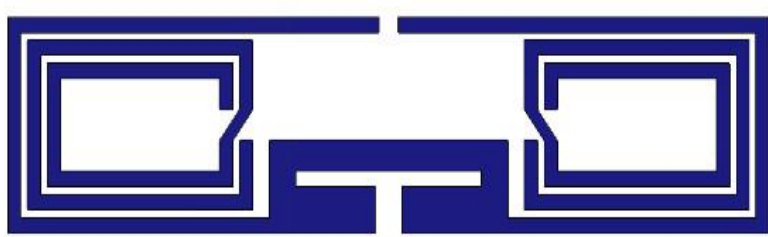

(b)

Figure.8 Proposed antenna: (a) without metamaterial and (b) with metamaterial

Table 2. Dimensions of the proposed antenna

\begin{tabular}{|c|c|}
\hline Parameter & Value $(\mathbf{m m})$ \\
\hline Lp & 17 \\
\hline Wp & 11 \\
\hline Lf & 2 \\
\hline Wf & 1 \\
\hline
\end{tabular}


The simulation results in terms of the reflection coefficient of the proposed reader antenna with and without metamaterials are presented in Fig. 9. It can be noticed that without metamaterials the antenna resonates at the frequency $1.13 \mathrm{GHz}$ and with the integration of the spiral SRR cells, the resonance frequency has shifted to the $868 \mathrm{MHz}$ frequency which is standardized for RFID applications in Europe and Morocco. This mean that the use of the spiral SRR cells allowed us to miniaturize the proposed antenna by $72.81 \%$. It is also observed that the impedance matching of the proposed antenna has been improved by the use of metamaterial cells from $-15 \mathrm{~dB}$ for the first antenna to $-30 \mathrm{~dB}$ for the final structure.

\subsection{Results and discussion}

After the design and optimization of the proposed antenna, we started the simulation using another electromagnetic software to validate the final structure, the comparison results obtained by ADS (Advanced Design System) and CST software are reported in Fig. 10. We can observe that there is a small difference between the obtained results, this difference is due to the different calculation methods used by both software.

The computed results in terms of gain versus frequency are illustrated in Fig. 11, as can be observed, the gain is between 1 and $2.2 \mathrm{~dB}$ for this antenna and equal to $1.69 \mathrm{~dB}$ around the central frequency

\section{Magnitude (dB)}

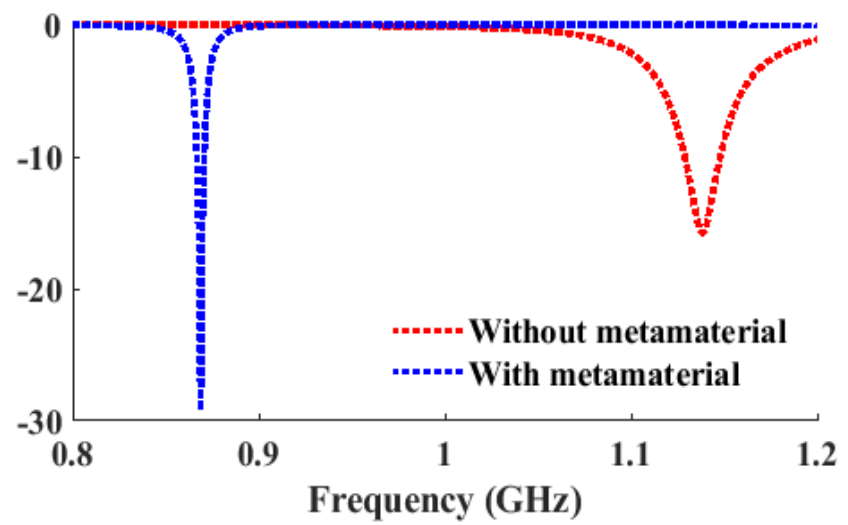

Figure.9 Comparison of the simulated reflection coefficient of the proposed antenna

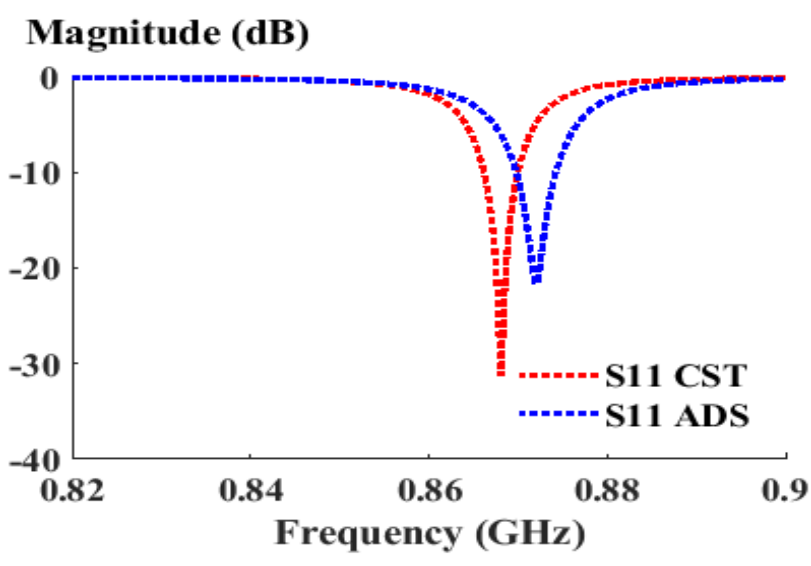

Figure.10 Comparison of the results obtained by CST and HFSS

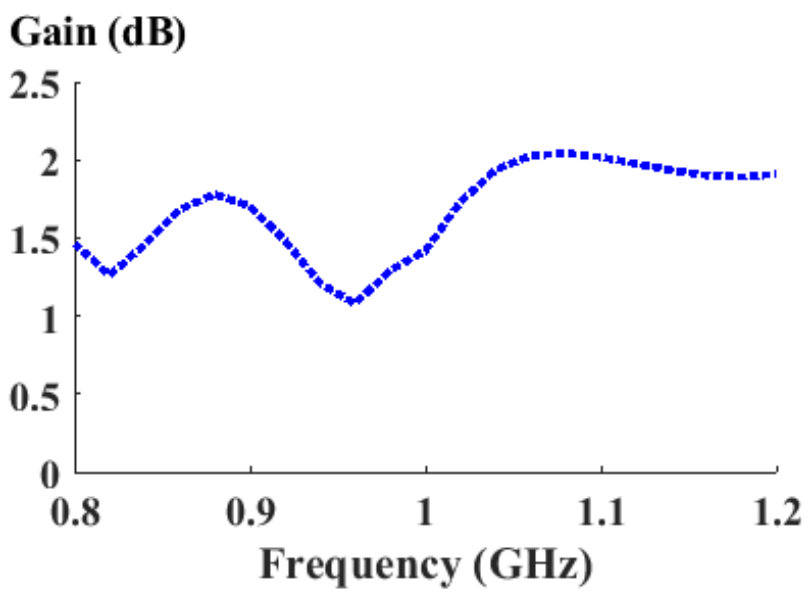

Figure.11 Gain versus frequency of the proposed antenna

The distribution of the surface currents is illustrated in Fig. 12, it is observed that the maximum of the current is around the two metamaterial cells.

The polar radiation patterns in the $\mathrm{H}$ and $\mathrm{E}$ planes is shown in Fig. 13, the antenna has an omnidirectional radiation.

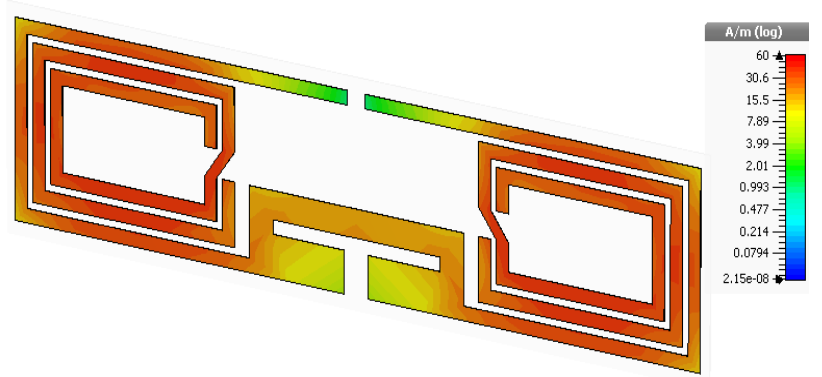

Figure.12 Current distribution of the proposed antenna 


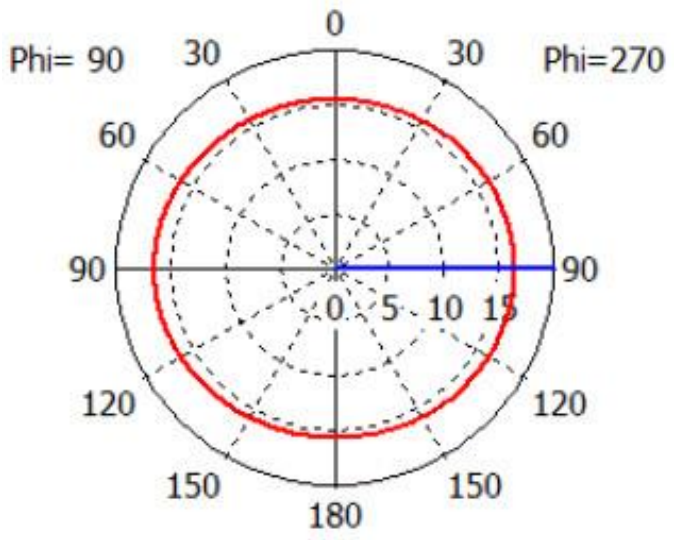

(a)

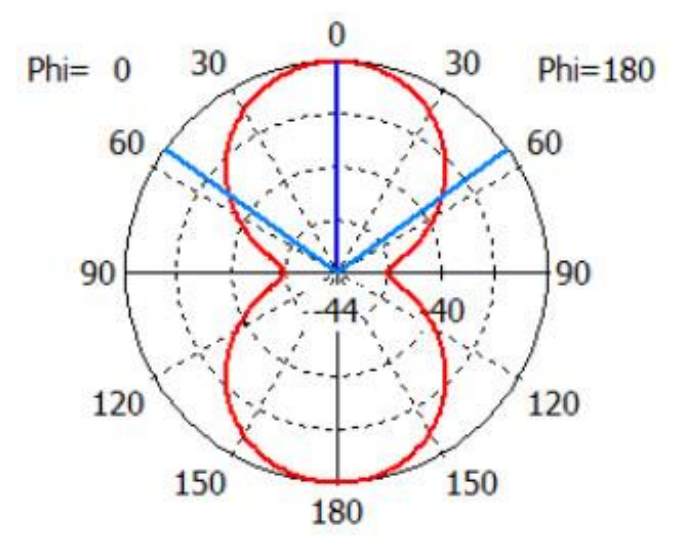

(b)

Figure.13 Radiation patterns of the proposed antenna at: (a) E-plane and (b) H-plane.

\section{Tag antenna design}

After the design of the RFID reader antenna which is adapted to $50 \mathrm{Ohms}$, in this part, we used the same antenna and we adjusted it to the input impedance of a commercial chip dedicated to passive RFID applications in the Moroccan and European UHF band.

The first section will be dedicated to the presentation of the RFID chip, the second section will be dedicated to the geometry of the proposed antenna and in the last section we will present the obtained results.

\subsection{RFID chip}

In this work, we have used a "Monza 1a" RFID chip which has an input impedance of $Z c=36-j 117 \Omega$ at $868 \mathrm{MHz}$. For a maximum power transfer, the impedance of the antenna $Z a$ to be designed must be equal to the conjugate of the impedance of the RFID chip $Z c$, it means that $Z a$ must be equal to $36+j 117$ $\Omega$. The RFID chip can be modeled electrically, by a series or parallel circuit as represented in Fig. 14.

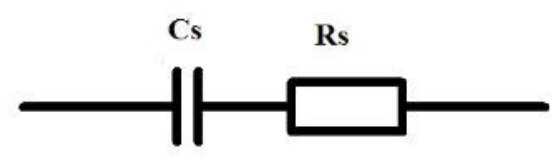

(a)

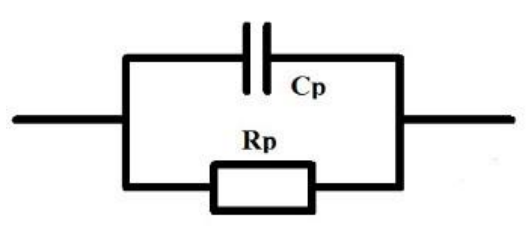

(b)

Figure.14 RFID model: (a) series circuit and (b) parallel circuit

The circuit parameters $R s, C s, R p$ and, $C p$ are calculated from the following equations [18]:

$$
\begin{aligned}
& R s=\operatorname{Re}(Z c) \\
& C s=\frac{1}{2 \cdot \pi \cdot f \cdot \operatorname{Im}(Z c)} \\
& R p=\frac{\operatorname{Re}(Z c)^{2}+\operatorname{Im}(Z c)^{2}}{\operatorname{Re}(Z c)} \\
& C p=\frac{\operatorname{Im}(Z c)}{2 \cdot \pi \cdot f \cdot\left(\operatorname{Im}(Z c)^{2}+\operatorname{Re}(Z c)^{2}\right)}
\end{aligned}
$$

Where $R s$ and $R p$ are the series and parallel resistors. $C s$ and $C p$ are the series and parallel capacitors respectively.

Fig. 15 shows the real and imaginary parts of the impedance of the chip Monza 1a versus the frequency obtained from the parallel model.

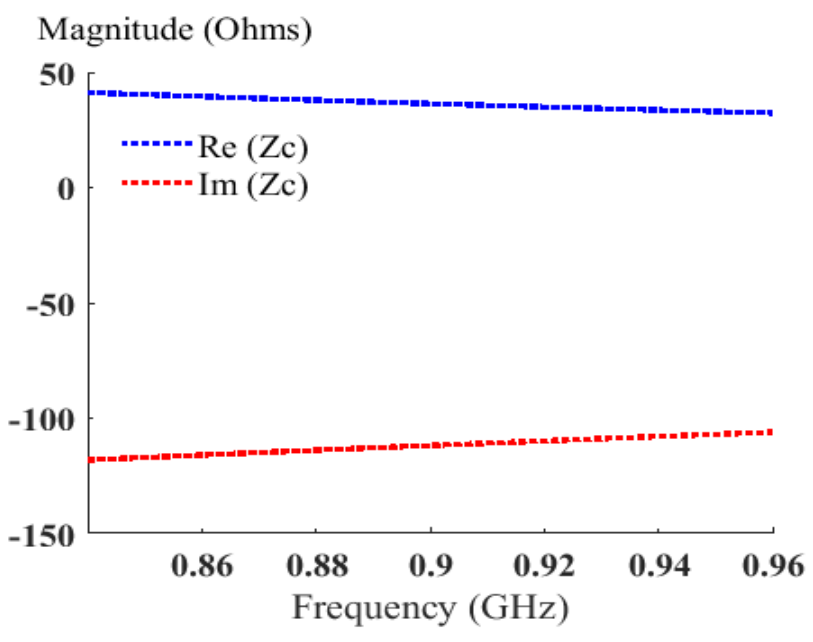

Figure.15 Impedance of the parallel model of the RFID chip "Monza 1a" 


\subsection{Tag antenna geometry}

We started designing the RFID tag antenna by using the antenna that we have designed for the RFID interrogator in the previous part.

The antenna has been adapted to the "Monza 1a" chip by a double T-Matching circuit as shown in Fig. 16. The antenna is simple, compact and designed on one side of a PET substrate having dielectric constant of 3.0 and loss tangent of 0.002. The optimized parameters of the final antenna are achieved by a parametric study on the double Tmatching structure. The total size of the proposed tag antenna is about $63 \mathrm{~mm} \times 14 \mathrm{~mm}$.

\subsection{Results and discussion}

Fig. 17 presents the proposed tag antenna input impedance against frequency obtained by CST. These results were obtained by using a parametric study on certain parameters of the double $\mathrm{T}$ matching circuit. We have noticed that some parameters affect the real part of the input impedance, some parameters affect the imaginary part and others affect both parts. After this study, we have optimized the antenna to reach a miniature size, a good matching impedance, a good gain and a good reading distance. From Fig. 17, we can observe that at the resonance frequency which is $868 \mathrm{MHz}$, the input impedance of the antenna corresponds to the conjugate of the impedance of the RFID chip "Monza 1a". Indeed, we obtained a complex impedance $Z a=33+j 114 \Omega$ which is close to the conjugate of the impedance of the RFID chip $Z c$.

The calculated results are validated by another electromagnetic software ADS as presented in the same figure, we can observe that there is a good agreement between both results.

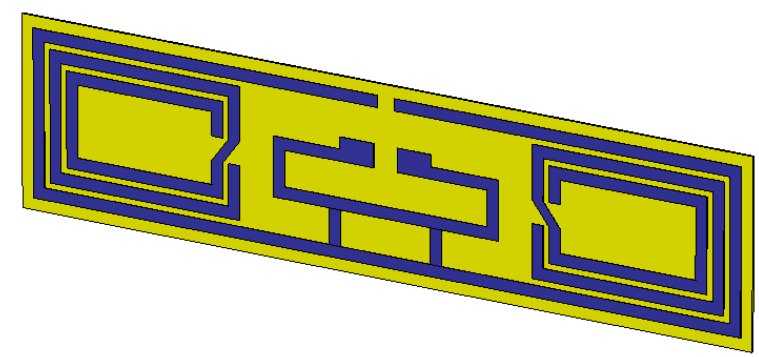

Figure.16 Proposed antenna for RFID Tag
The reflection coefficient of the proposed tag is reported in Fig. 18, we have obtained a good impedance matching of $-24 \mathrm{~dB}$ at the frequency of $868 \mathrm{MHz}$. To validate our structure, we launched another simulation under the ADS software and we obtained a good agreement between the results obtained by ADS and the results obtained by CST.

The gain against frequency of the proposed tag antenna is illustrated in Fig. 19, we can notice that the antenna has a positive gain over the entire frequency band and equal to $2.2 \mathrm{dBi}$ at the resonance frequency.

The radiation patterns of this antenna at $\mathrm{E}$ and $\mathrm{H}$ planes is reported in Fig. 20, it is noticed that the antenna has an omnidirectional radiating which is suitable for RFID applications.

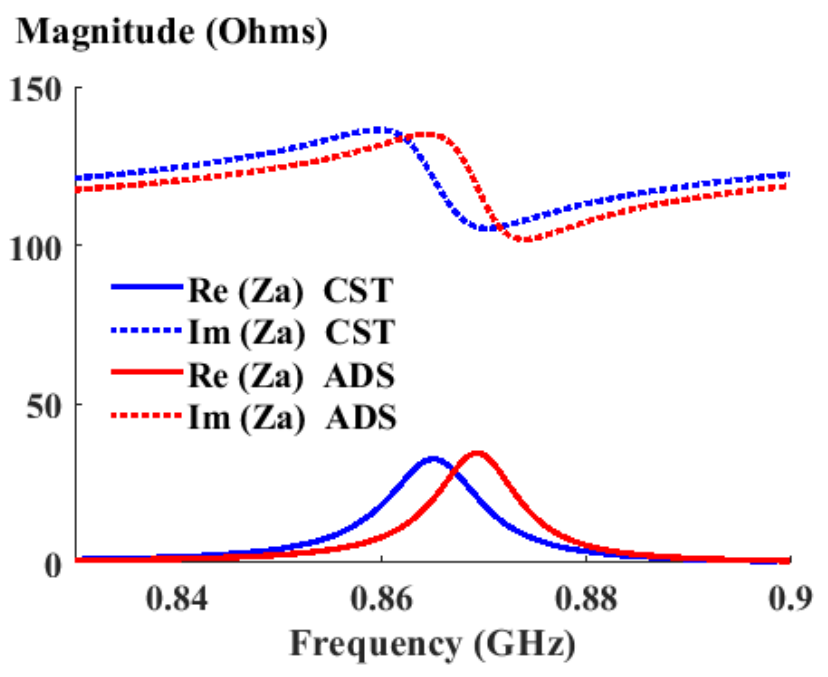

Figure.17 Comparison of the input impedance of the proposed tag antenna

Magnitude (dB)

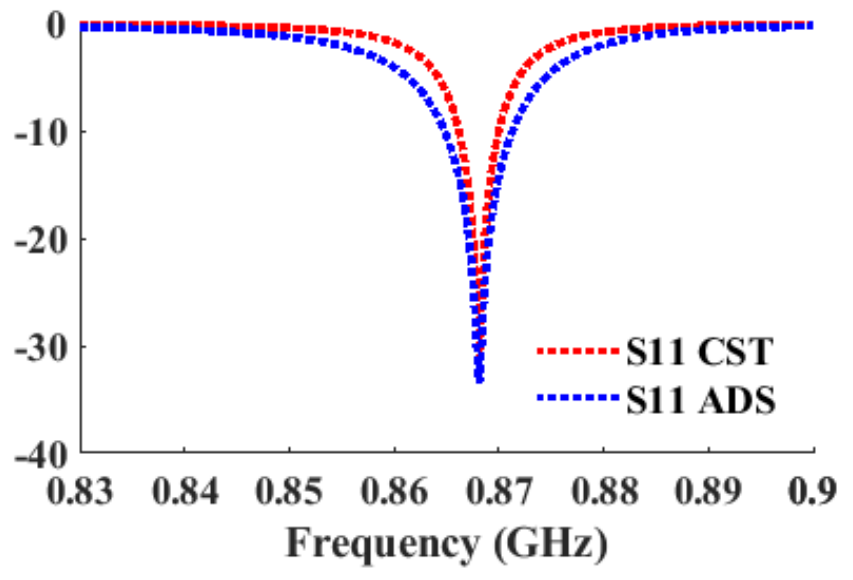

Figure.18 Coefficient reflection of the proposed tag antenna 


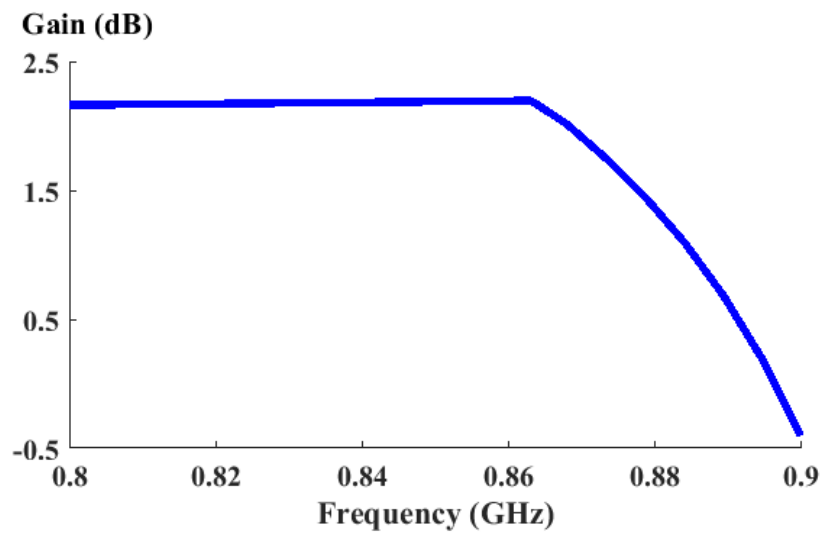

Figure.19 Gain against frequency of the proposed tag antenna

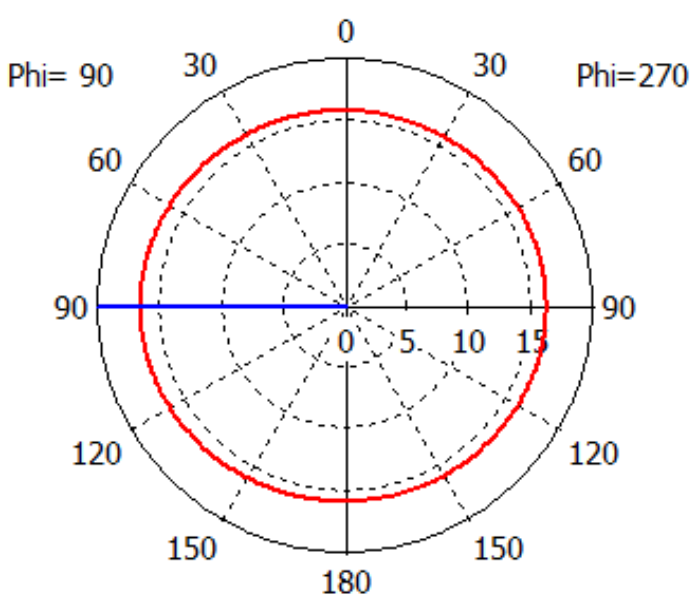

(a)

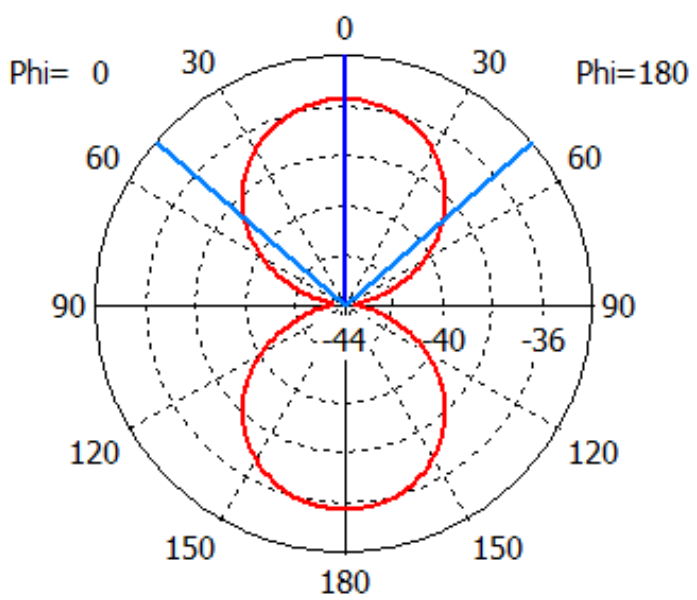

(b)

Figure.20 Radiation patterns of the proposed tag antenna at: (a) E-plane and (b) H-plane

The currents surface distribution is reported in Fig 21, it is noticed that the maximum current is around the double T-matching circuit and the metamaterial unit cells.

Another important parameter of an RFID antenna is the reading distance. It determines the distance from which the antenna can receive and transmit data. The maximum reading distance depends on several parameters such as the sensitivity threshold of the chip Pth, the gain of the antenna Gt, the transmission coefficient $\tau$, the velocity of the light in the vacuum $\mathrm{c}$ and the isotropic power EIRP, it is given by the following equation [19]:

$$
D \max =\frac{c}{4 . \pi \cdot f} \sqrt{\frac{E I R P \cdot \tau \cdot G t}{P t h}}
$$

Were $\tau$ is related to input impedance of the antenna $\mathrm{Za}$ and the impedance of the microchip by:

$$
\tau=\frac{4 \cdot R a \cdot R c}{|Z a+Z c|}
$$

The reading distance of the proposed antenna is around $6.3 \mathrm{~m}$ at the resonance frequency, it is reported in Fig. 22.

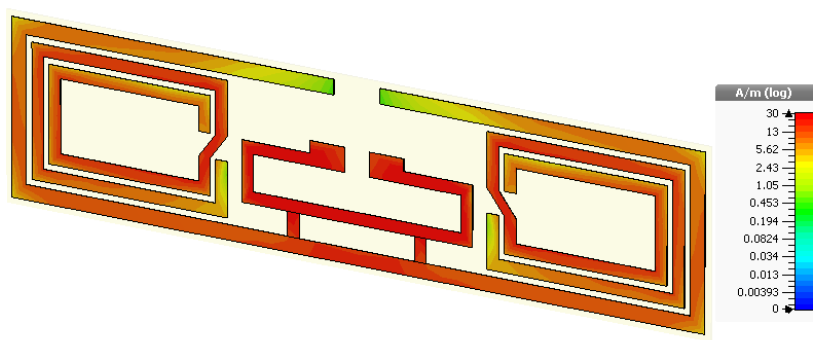

Figure.21 Surface current distribution of the proposed tag antenna

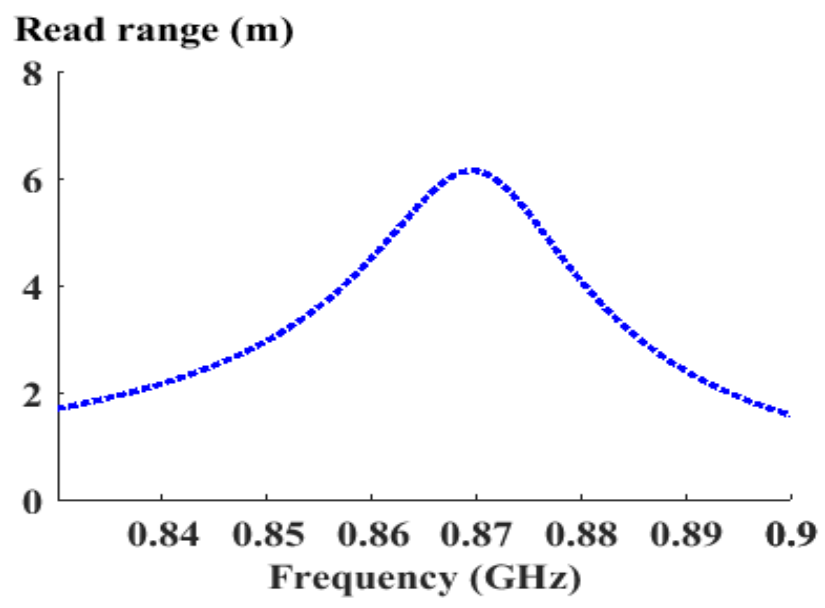

Figure.22 Read range of the proposed tag antenna 
After this validation, we have conducted to a comparison between our proposed structure and some published works at the UHF RFID band in terms of size, total area occupied by the antenna, gain and, read range in free space, the comparison results are reported in Table 3. From this result, we can conclude that we reached a miniaturization of $82.06 \%$ with good performances in terms of gain and read range.

\section{Conclusion}

In this work, we presented a dipole antenna for RFID applications based on metamaterials. In a first step, we have adjusted the antenna to an impedance of 50 Ohms for integration into an RFID base station. The use of the spiral SRR metamaterial unit cells allowed us to miniaturize the total size of the proposed antenna by $72.81 \%$. In a second step, the antenna has been adjusted to a commercial RFID microchip which has a complex impedance. For further matching the proposed tag antenna with the Monza 1a chip a double T-matching technique has been used. We have reached a miniaturization rate of $82.06 \%$ in comparison with the conventionnel tag antenna. The proposed RFID antenna presents a good performance in terms of return loss, gain, and, reading range. In our future work, we will study the effect of metamaterials on the ground plane of an antenna which will be used for the patient's identification.

\section{Acknowledgments}

We thank Mr. Mohamed LATRACH Professor in ESEO, Engineering Institute in Angers, France, for allowing us to use all the equipment's and EM solvers available in his laboratory.

Table 3. Comparison of the proposed antenna with some published work

\begin{tabular}{|c|c|c|c|c|}
\hline Ref & $\begin{array}{c}\text { Size } \\
\left(\mathbf{m m}^{2}\right)\end{array}$ & $\begin{array}{c}\text { Total } \\
\text { area } \\
\left(\mathbf{m m}^{2}\right)\end{array}$ & $\begin{array}{c}\text { Gain } \\
(\mathbf{d B})\end{array}$ & $\begin{array}{c}\text { Read } \\
\text { rang } \\
(\mathbf{m})\end{array}$ \\
\hline$[4]$ & $74 \times 28$ & 2072 & 1.6 & 5.7 \\
\hline$[6]$ & $78 \times 30$ & 2340 & 1.11 & 5 \\
\hline$[8]$ & $48 \times 22$ & 1056 & -1.39 & - \\
\hline$[20]$ & $60 \times 40$ & 2400 & 2 & - \\
\hline$[21]$ & $91 \times 36$ & 3276 & 5.2 & - \\
\hline Our work & $63 \times 14$ & 882 & 2.2 & 6.3 \\
\hline
\end{tabular}

\section{References}

[1] K. Eguchi, I. oota, S. Terada, and H. Zhu, "2x/3x step-up switched-capacitor (SC) AC-DC converters for RFID tags", International Journal of Intelligent Engineering and Systems, Vol.4, No.1, pp.1-9, 2011.

[2] H. Tizyi, F. Riouch, A. Tribak, A. Najid, and A. Mediavilla, "CPW and Microstrip Line-Fed Compact Fractal Antenna for UWB-RFID Applications", Progress in Electromagnetics Research C, Vol.65, pp.201-209, 2016.

[3] M. Z. B. Ahmed, M. R. E. Fekar, N. SeladjiHassaine, and F. Z. Marouf, "Analysis of Patch and Printed Antennas integrated into UHF RFID Passive Tags", International Journal of Microwave and Optical Technology, Vol.6, No.11, pp.460-468, 2016.

[4] S. K. Goudos, K. Siakavara, and J. N. Sahalos, "Novel Spiral Antennas Design Using Swarm Intelligence for Passive UHF RFID Tags", In: Proc. of IEEE European Conference on Antennas and Propagation, Gothenburg, Sweden, pp.3223-3227, 2013.

[5] H. Kimouche and H. Zemmour, "A Compact Fractal Dipole Antenna For $915 \mathrm{MHz}$ and 2.4 GHz RFID Tag Applications", Progress in Electromagnetics Research Letters, Vol.26, pp.105-114, 2011.

[6] A. Elhamraoui, E. Abdelmounim, A. Errkik, H. Bennis and M. Latrach, "A New Design of a Miniature RFID Tag Antenna for Metallic Objects”, In: Proc. of International Conference on Computing and Wireless Communication Systems, Larache, Morocco, pp.1-5, 2017.

[7] A. Choudhary, K. Gopal, D. Sood, and C. Charu Tripathi, "Development of compact inductive coupled meander line RFID tag for near-field applications", International Journal of Microwave and Wireless Technologies, Vol.9, No.4, pp.757-764, 2017.

[8] I. Frigui, M. Karoui, H. Ghariani, and M. Lahiani, "New Folded Tag Antenna with Chanel Shaped Metamaterial", International Journal on Communications Antenna and Propagation, Vol.6, No.6, pp. 375-380, 2016.

[9] B. Nasiri, A. Errkik, J. Zbitou, A. Tajmouati, L. Elabdellaoui, and M. Latrach, "A Miniature Microstrip BSF Using Complementary Split Ring Resonator", International Journal of Intelligent Engineering and Systems, Vol.11, No.3, pp.29-36, 2018.

[10] A. Kumar, N. Kumar, and C. Gupta, "Review on Microstrip Patch Antennas using Metamaterials", International Journal of 
Engineering Research and General Science, Vol.2, No.4, pp.678-682, 2014.

[11] A. Ennajih, J. Zbitou, M. Latrach, A. Errkik, L. El Abdellaoui, and A. Tajmouati, "Dual Band Metamaterial Printed Antenna Based on CSRR For RFID Applications", International Journal of Microwave and Optical Technology, Vol.12, No.2, pp.106-113, 2017.

[12] P. K. Choudhary and R. K. Chaudhary, "A Compact Via-Less Metamaterial Wideband Bandpass Filter Using Split Circular Rings and Rectangular Stub", Progress in Electromagnetics Research Letters, Vol.72, pp.99-106, 2018.

[13] B. Nasiri, A. Errkik, J. Zbitou, A. Tajmouati, L. El Abdellaoui, and M. Latrach, "A Compact Miniature Microstrip Low pass Filter Based on Dumbbell DGS and SRR Unit Cell", International Journal of Microwave and Optical Technology, Vol.13, No.1, pp.32-39, 2018.

[14] A. Kumar, J. Mohan and H. Gupta, "Novel Metamaterials with their Applications to Microstrip Antenna", International Journal of Microwave and Optical Technology, Vol.11, No.3, pp.188-195, 2016.

[15] M. Boddapati, and P. Kishore, "Bandwidth Enhancement of CPW-Fed Elliptical Curved Antenna with Square SRR", International Journal of Intelligent Engineering and Systems, Vol.11, No.2, pp.68-75, 2018.

[16] M. Soliman, M. Dwairi, I. Sulayman, and S. Almalki, "A Comparative Study for Designing and Modeling Patch Antenna with Different Electromagnetic CAD Approaches", International Journal on Communications Antenna and Propagation, Vol.6, No.2, pp.9095, 2016.

[17] A. Constantine Balanis, Antenna Theory and Design, Second Edition., John Wiley \& Sons, Inc., 1997.

[18] A. Ghiotto, S. F. Cantalice, T. P. Vuong, A. Pouzin, G. Fontgalland, and S. Tedjini, "Miniaturized patch antenna for the Radio Frequency Identification of metallic objects", In: Proc. of IEEE MTT-S International Microwave Symposium Digest, Atlanta, USA, pp.583-586, 2008.

[19] A. Mohd Faudzi, M. Tarmizi Ali, I. Ismail, H. Jumaat, and N. Hidayah Mohd Sukaimi, "Metal Mountable Ladder Feed Line UHFRFID Tag Antenna", International Journal of Electrical and Computer Engineering, Vol.5, No.4, pp.750-758, 2015.
[20] A. Sofi, K. Roky, M. Bouhorma, and I. H. Baraka, "Novel Antennas for UHF RFID Tags: Design and Miniaturization", International Journal of Electrical and Computer Engineering, Vol.4, No.1, pp.75-80, 2014.

[21] T. Andriamiharivolamena, and S. Tajdjini, "Compact and Small Size Antenna for an UHF RFID Tag placed over a metallic Thing", In: Proc. of the $32^{\text {nd }}$ URSI GASS, Montreal, Canada, pp.1-3, 2017. 